Article

\title{
Symmetry Group Classification and Conservation Laws of the Nonlinear Fractional Diffusion Equation with the Riesz Potential
}

\author{
Nikita S. Belevtsov ${ }^{\dagger}$, Stanislav Yu. Lukashchuk *,+ \\ Laboratory "Group Analysis of Mathematical Models in Natural and Engineering Sciences", \\ Ufa State Aviation Technical University, Ufa 450008, Russia; nikitabelewtsov@mail.ru \\ * Correspondence: lsu@ugatu.su \\ t These authors contributed equally to this work.
}

Received: 24 December 2019; Accepted: 16 January 2020; Published: 19 January 2020

check for updates

\begin{abstract}
Symmetry properties of a nonlinear two-dimensional space-fractional diffusion equation with the Riesz potential of the order $\alpha \in(0,1)$ are studied. Lie point symmetry group classification of this equation is performed with respect to diffusivity function. To construct conservation laws for the considered equation, the concept of nonlinear self-adjointness is adopted to a certain class of space-fractional differential equations with the Riesz potential. It is proved that the equation in question is nonlinearly self-adjoint. An extension of Ibragimov's constructive algorithm for finding conservation laws is proposed, and the corresponding Noether operators for fractional differential equations with the Riesz potential are presented in an explicit form. To illustrate the proposed approach, conservation laws for the considered nonlinear space-fractional diffusion equation are constructed by using its Lie point symmetries.
\end{abstract}

Keywords: space-fractional filtration equation; Riesz potential; Lie point symmetry group; group classification; nonlinear self-adjointness; conservation laws

\section{Introduction}

Fractional differential equations (FDEs) with multi-dimensional spatial fractional differential operators have attracted considerable attention during the last decade due to the possibility to describe power-law long-range interactions in complex systems [1-3]. In particular, such equations can be efficiently used for modelling a fluid flow in naturally fractured porous media, which is a very important problem for the oil industry. Examples of such fractional differential models can be found in $[4,5]$. Usually, the integer-order derivatives of the Riesz potential or fractional Laplacian (see, e.g., [6,7]) are used as fractional differential operators in these models. These fractional operators are well studied [7-9] and have a lot of similar properties. Nevertheless, there are some differences in classes of functions for which these operators exist. As a result, FDEs corresponding to these two types of fractional spatial operators will also have different qualitative properties (see, e.g., discussion in [10]). Note that nowadays FDEs with the Riesz potential are much less studied than FDEs containing fractional Laplacian. Therefore, in this paper, we restrict our attention by equations with the Riesz potential.

Finding exact solutions to nonlinear space-fractional FDEs is a sufficiently complex problem. Nevertheless, this problem can be significantly simplified if symmetry properties of the considered equation are known. These properties can be studied by the methods of Lie group analysis of differential equations [11-13]. Recently, some basic Lie symmetry group methods have been extended to fractional differential equations with the Riemann-Liouville and Caputo fractional derivatives 
(see the survey papers [14,15] and references therein). In [16], the algorithm for finding the Lie point symmetry group of FDEs with the Riesz potential was firstly proposed, and symmetries of the linear two-dimensional space-fractional filtration equation with the Riesz potential were obtained.

Classification of nonlinear equations belonging to a certain class with respect to symmetry groups is an important task of modern Lie group analysis. Fundamentals of the group classification originated in works by Sophus Lie. An efficient approach to symmetry group classification was developed by Ovsyannikov [17] (see also [11]). He first performed a complete group classification of the nonlinear heat equation with the thermal conductivity treated as a function of the temperature. In [18], the group classification of time-fractional analogues of this equation with the Riemann-Liouville and Caputo fractional derivatives was performed. Nevertheless, the problem of group classification for the space-fractional FDEs with the Riesz potential has never been considered. In this paper, we present the results of Lie point symmetry group classification for a nonlinear space-fractional diffusion equation containing the Riesz potential with respect to diffusivity function.

It is well known that there is a close connection between symmetries and conservation laws (see, e.g., [19,20]). In 1918, Emmy Noether proved [21] that a conservation law can be obtained by the group invariance principle from the variational integral with a Lagrangian function as an integrand. An efficient constructive algorithm for finding conservation laws of a differential equation possesses a Lagrangian was proposed by Ibragimov and can be found in [22]. Recently, this algorithm was enhanced to fractional differential equations with Lagrangians depending on fractional differential variables formed by arbitrary compositions of fractional integral operators and integer-order differential operators [23].

To find conservation laws for differential equations without Lagrangians, the general concept of nonlinear self-adjointness of integer-order differential equations was proposed by Ibragimov [24-26]. He also proved that the constructive algorithm proposed earlier is also applicable to find conservation laws for nonlinear self-adjoint equations with formal Lagrangians. In [27-29], it is shown that the concept of nonlinear self-adjointness can be enhanced to FDEs with the Riemann-Liouville and Caputo fractional derivatives. In this paper, we extend this approach to FDEs with the Riesz potential.

The paper is organized as follows. In Section 2, we recall necessary definitions of the Lie symmetry group theory and give essential theorems on the Riesz potential properties. In Section 3, the results of group classification are presented for a nonlinear space-fractional diffusion equation with the Riesz potential. Section 4 is devoted to considering the nonlinear self-adjointness of FDEs containing the Riesz potential. In Section 5, we present a technique of finding conservation laws for such equations using their symmetries.

\section{Preliminaries}

This section provides a brief introduction to the basic principles of Lie group analysis and its application to fractional differential equations with the Riesz potential. First of all, we give necessary definitions.

Let $x^{0} \in[0, T](T>0)$ and $x=\left(x^{1}, \ldots, x^{n}\right) \in R^{n}$ be the time variable and the vector of spatial variables, respectively. The function $u=u\left(x^{0}, x\right)$ will be considered as a dependent variable.

We will deal with one-parameter Lie groups of point transformations [13] given by

$$
\begin{array}{ll}
\bar{x}^{i}=f^{i}\left(x^{0}, x, u, a\right), & \left.f^{i}\right|_{a=0}=x^{i}, \quad i=0,1, \ldots, n ; \\
\bar{u}=g\left(x^{0}, x, u, a\right), & \left.g\right|_{a=0}=u,
\end{array}
$$

depending on a continuous parameter $a$. The infinitesimal transformations of group (1) can be written as

$$
\bar{x}^{i} \approx x^{i}+a \xi^{i}\left(x^{0}, x, u\right), \quad \bar{u} \approx u+a \eta\left(x^{0}, x, u\right),
$$


where

$$
\xi^{i}\left(x^{0}, x, u\right)=\left.\frac{\partial f^{i}\left(x^{0}, x, u, a\right)}{\partial a}\right|_{a=0^{\prime}} \quad \eta\left(x^{0}, x, u\right)=\left.\frac{\partial g\left(x^{0}, x, u, a\right)}{\partial a}\right|_{a=0} .
$$

The approximate equality $f \approx g$ means that $f=g+o(a)$.

The infinitesimal generator of group (1) is the linear first-order differential operator

$$
X=\xi^{i}\left(x^{0}, x, u\right) \frac{\partial}{\partial x^{i}}+\eta\left(x^{0}, x, u\right) \frac{\partial}{\partial u} .
$$

Summation over repeated indices is implied in this paper.

Any group (1) with

$$
\xi^{i}=\xi^{i}\left(x^{0}, x\right), \quad \eta\left(x^{0}, x, u\right)=\eta^{0}\left(x^{0}, x\right)+\eta^{1}\left(x^{0}, x\right) u
$$

is called a linear autonomous one-parameter Lie group of point transformations [30] (see also [14,15]).

The Riesz potential [7] in $n$-dimensional space is defined by

$$
R^{\alpha} u\left(x^{0}, x\right)=\frac{1}{\gamma_{n}(\alpha)} \int_{R^{n}} \frac{u\left(x^{0}, \mu\right)}{|x-\mu|^{n-\alpha}} d \mu,
$$

where $\mu=\left(\mu^{1}, \ldots, \mu^{n}\right)$ and

$$
\gamma_{n}(\alpha)=2^{\alpha} \pi^{n / 2} \Gamma\left(\frac{\alpha}{2}\right) / \Gamma\left(\frac{n-\alpha}{2}\right) .
$$

Remark 1. In a 1D case, the Riesz potential can be rewritten as a sum of the left-sided $I_{+}^{\alpha} u$ and the right-sided $I_{-}^{\alpha} u$ Liouville fractional integrals on $R$ :

$$
R^{\alpha} u\left(x^{0}, x\right)=\frac{\Gamma(\alpha)}{\gamma_{n}(\alpha)}\left(I_{+}^{\alpha} u+I_{-}^{\alpha} u\right), \quad x \in R,
$$

where

$$
I_{+}^{\alpha} u=\frac{1}{\Gamma(\alpha)} \int_{-\infty}^{x} \frac{u\left(x^{0}, \mu\right)}{(x-\mu)^{1-\alpha}} d \mu, \quad I_{-}^{\alpha} u=\frac{1}{\Gamma(\alpha)} \int_{x}^{\infty} \frac{u\left(x^{0}, \mu\right)}{(\mu-x)^{1-\alpha}} d \mu .
$$

In addition, in [31], it was proved that, if $u$ is a radial function, i.e., $u=u\left(x^{0},|x|\right)$, then the Riesz potential can be written as

$$
R^{\alpha} u\left(x^{0}, x\right)=\left.2^{-\alpha}|x|^{2-n}\left(I_{0+}^{\frac{\alpha}{2}} s^{\frac{n-\alpha}{2}-1} I_{-}^{\frac{\alpha}{2}} u\left(x^{0}, \sqrt{s}\right)\right)\right|_{s=x^{2}} .
$$

In special cases corresponding to representations (6) and (7), equations with the integer-order derivatives of the Riesz potential are equivalent to fractional differential equations with the Riemann-Liouville fractional derivatives. Such equations will not be considered in this paper.

A group (1) can be prolonged to the Riesz potential (5). The corresponding prolongation formula is given by the following theorem.

Theorem 1 ([16]). The infinitesimal transformation of the Riesz potential (5) induced by the group (1) has the form

$$
\bar{R}^{\alpha} \bar{u} \approx R^{\alpha} u+a \zeta^{\alpha},
$$

where $\zeta^{\alpha}$ is given by the prolongation formula

$$
\zeta^{\alpha}=R^{\alpha}\left(\eta-\xi^{i} u_{i}\right)+\xi^{i} D_{i}\left(R^{\alpha} u\right)
$$

Here, $\bar{R}^{\alpha}$ is the Riesz operator with respect to $\bar{x}, u_{i}=\frac{\partial u}{\partial x^{i}}$, and $D_{i}$ denotes the operator of total differentiation with respect to $x^{i}$. 
We will consider fractional differential equations with integer-order derivatives of the Riesz potential (5). If such equation does not change the form when it is written in the new variables $\bar{x}^{i}$ $(i=0,1, \ldots, n)$ and $\bar{u}$ defined by group (1), then the corresponding one-parameter group is called Lie symmetry group of point transformations for this equation. The infinitesimal generator of such a group is called an infinitesimal symmetry of this equation.

By using transformation (8), the prolongation formula for any integer-order derivative of the Riesz potential can be constructed. We introduce the notation

$$
\left(R^{\alpha} u\right)_{i_{1} \ldots i_{s}}=\frac{\partial^{s}\left(R^{\alpha} u\right)}{\partial x^{i_{1}} \ldots \partial x^{i_{s}}}
$$

where $i_{1}, \ldots, i_{s}=0,1, \ldots, n$.

Theorem 2. The infinitesimal transformation of $\left(R^{\alpha} u\right)_{i_{1} \ldots i_{s}}$ induced by the group (1) can be written as

$$
\left(\bar{R}^{\alpha} \bar{u}\right)_{i_{1} \ldots i_{s}} \approx\left(R^{\alpha} u\right)_{i_{1} \ldots i_{s}}+a \zeta_{i_{1} \ldots i_{s}}^{\alpha}
$$

where $\zeta_{i_{1} \ldots i_{s}}^{\alpha}$ is given by the prolongation formula

$$
\zeta_{i_{1} \ldots i_{s}}^{\alpha}=D_{i_{s}} \ldots D_{i_{1}} R^{\alpha}\left(\eta-\xi^{i} u_{i}\right)+\xi^{i} D_{i}\left(R^{\alpha} u\right)_{i_{1} \ldots i_{s}} .
$$

Let $F=0$ be a fractional differential equation with the Riesz potential. We denote by $\tilde{X}$ an infinitesimal generator of the Lie group of point transformations prolonged to all integer-order and fractional-order differential variables included into the function $F$. Then, the necessary condition of $X$ to be a symmetry of the equation $F=0$ can be written as

$$
\left.(\tilde{X} F)\right|_{F=0}=0 .
$$

Note that, contrary to integer-order differential equations, the invariance condition (11) is a necessary but not sufficient condition for fractional differential equations (the detailed discussion of this fact can be found in [14]).

Now we give some useful properties of the Riesz potential in two-dimensional space. We introduce the integral operator $I_{\beta}^{m, n}$ by

$$
I_{\beta}^{m, n} f(x, y)=\frac{1}{\gamma_{2}(\alpha)} \int_{-\infty}^{\infty} \frac{(\mu-x)^{m}(v-y)^{n} f(\mu, v)}{\left[(\mu-x)^{2}+(v-y)^{2}\right]^{\frac{\beta}{2}}} d \mu d v .
$$

Proposition 1 ([16]). The integral operator (12) possesses the property

$$
I_{4-\alpha}^{m+2, n}=I_{2-\alpha}^{m, n}-I_{4-\alpha}^{m, n+2} .
$$

The following theorem gives a generalization of the Leibniz rule for the two-dimensional Riesz potential [16].

Theorem 3. Let $f(x, y)$ be an analytic function in $R^{2}$ and $g(x, y)$ be a function such that integrals $I_{2-\alpha}^{m, n} g$ exist for any $m, n \in N \cup\{0\}$. Then,

$$
R^{\alpha}(f g)=\sum_{m=0}^{\infty} \sum_{n=0}^{\infty} \frac{1}{m ! n !} \frac{\partial^{m+n} f}{\partial x^{m} \partial y^{n}} I_{2-\alpha}^{m, n} g .
$$

From Theorem 3 and the identity (13), we infer the following. 
Corollary 1. Under assumptions of Theorem 3, the following equalities hold:

$$
D_{x}^{2} R^{\alpha}(f g)=\sum_{m=0}^{\infty} \sum_{n=0}^{\infty} \frac{\partial^{m+n} f}{\partial x^{m} \partial y^{n}} J_{x, 2-\alpha}^{m, n}, \quad D_{y}^{2} R^{\alpha}(f g)=\sum_{m=0}^{\infty} \sum_{n=0}^{\infty} \frac{\partial^{m+n} f}{\partial x^{m} \partial y^{n}} J_{y, 2-\alpha}^{m, n},
$$

where

$$
\begin{aligned}
& J_{x, 2-\alpha}^{m, n}=\frac{1}{m ! n !}\left(m(m-1) I_{2-\alpha}^{m-2, n}+2 m D_{x} I_{2-\alpha}^{m-1, n}+D_{x}^{2} I_{2-\alpha}^{m, n}\right), \\
& J_{y, 2-\alpha}^{m, n}=\frac{1}{m ! n !}\left(n(n-1) I_{2-\alpha}^{m, n-2}+2 n D_{y} I_{2-\alpha}^{m, n-1}+D_{y}^{2} I_{2-\alpha}^{m, n}\right) .
\end{aligned}
$$

\section{Group Classification of the Nonlinear Space-Fractional Porous Medium Equation}

In [16], it was shown that one phase flow of viscous compressible fluid through a naturally fractured oil reservoir can be modeled by a diffusion-type fractional differential equation with the Riesz potential. If the viscosity of fluid depends on pressure, then this equation becomes nonlinear. In this paper, we restrict our attention by the two-dimensional case. For convenience, we denote $x^{0}=t$, $x^{1}=x, x^{2}=y$. Then, the equation in question has the form

$$
u_{t}=\nabla\left(k(u) \nabla R^{\alpha} u\right),
$$

where $u=u(t, x, y), t>0,(x, y) \in R^{2}$, and $\alpha \in(0,1)$. In the linear case $(k(u)=1)$, the symmetry properties of this equation has been investigated in [16]. In this paper, a group classification of Equation (16) with respect to the function $k(u)$ is performed.

We note that, if $\alpha=0$, then Equation (16) coincides with the classical nonlinear heat equation. The group classification of this integer-order partial differential equation is well-known and can be found in [11]. It is very important that this classical nonlinear heat equation has only linear autonomous symmetries for any $k(u)$. Numerous calculations show that, if an integer-order partial differential equation has only linear autonomous symmetries, then the related partial fractional differential equation inherits this property. Therefore, we will perform group classification of (16) with respect to Lie point linear autonomous symmetries. The corresponding group generator has the form

$$
X=\xi^{0} \frac{\partial}{\partial t}+\xi^{1} \frac{\partial}{\partial x}+\xi^{2} \frac{\partial}{\partial y}+\eta \frac{\partial}{\partial u}
$$

with

$$
\xi^{0}=\xi^{0}(t, x, y), \quad \xi^{1}=\xi^{1}(t, x, y), \quad \xi^{2}=\xi^{2}(t, x, y), \quad \eta=\eta^{0}(t, x, y)+\eta^{1}(t, x, y) u .
$$

It can be easily verified that Equation (16) admits the following five-parameter group of equivalence transformations:

$$
\bar{u}=B_{1} u, \quad \bar{t}=B_{2} t+B_{1}, \quad \bar{k}=B_{2}^{-1} B_{3}^{2-\alpha} k, \quad \bar{x}=B_{3} x+B_{4}, \quad \bar{y}=B_{3} x+B_{5} .
$$

These transformations preserve the fractional differential structure of Equation (16) but change the form of classifying function $k(u)$. The symmetry group classification of Equation (16) will be performed up to these transformations.

For convenience, we rewrite Equation (16) as

$$
u_{t}=k(u)\left[D_{x}^{2}\left(R^{\alpha} u\right)+D_{y}^{2}\left(R^{\alpha} u\right)\right]+k^{\prime}(u) u_{x} D_{x}\left(R^{\alpha} u\right)+k^{\prime}(u) u_{y} D_{y}\left(R^{\alpha} u\right) .
$$


It can be seen that the prolongation of the generator (17) to all derivatives included in (16) has the form

$$
\begin{aligned}
& \bar{X}=\xi^{0} \frac{\partial}{\partial t}+\xi^{1} \frac{\partial}{\partial x}+\xi^{2} \frac{\partial}{\partial y}+\eta \frac{\partial}{\partial u}+\zeta_{t} \frac{\partial}{\partial u_{t}}+\zeta_{x} \frac{\partial}{\partial u_{x}}+\zeta_{y} \frac{\partial}{\partial u_{y}} \\
& +\zeta_{x}^{\alpha} \frac{\partial}{\partial D_{x} R^{\alpha} u}+\zeta_{y}^{\alpha} \frac{\partial}{\partial D_{y} R^{\alpha} u}+\zeta_{x x}^{\alpha} \frac{\partial}{\partial D_{x}^{2} R^{\alpha} u}+\zeta_{y y}^{\alpha} \frac{\partial}{\partial D_{y}^{2} R^{\alpha} u} .
\end{aligned}
$$

Here,

$$
\begin{aligned}
& \zeta_{t}=D_{t}\left(\eta-\xi^{0} u_{t}-\xi^{1} u_{x}-\xi^{2} u_{y}\right)+\xi^{0} u_{t t}+\xi^{1} u_{t x}+\xi^{2} u_{t y}, \\
& \zeta_{x}=D_{x}\left(\eta-\xi^{0} u_{t}-\xi^{1} u_{x}-\xi^{2} u_{y}\right)+\xi^{0} u_{t x}+\xi^{1} u_{x x}+\xi^{2} u_{y x}, \\
& \zeta_{y}=D_{y}\left(\eta-\xi^{0} u_{t}-\xi^{1} u_{x}-\xi^{2} u_{y}\right)+\xi^{0} u_{t y}+\xi^{1} u_{x y}+\xi^{2} u_{y y}
\end{aligned}
$$

and $\zeta_{x x}^{\alpha}, \zeta_{y y}^{\alpha}, \zeta_{x}^{\alpha}, \zeta_{y}^{\alpha}$ are obtained from (10) as

$$
\begin{aligned}
& \zeta_{x}^{\alpha}=D_{x} R^{\alpha}\left[\eta-\xi^{0} u_{t}-\xi^{1} u_{x}-\xi^{2} u_{y}\right]+\xi^{0} D_{x} D_{t}\left(R^{\alpha} u\right)+\xi^{1} D_{x}^{2}\left(R^{\alpha} u\right)+\xi^{2} D_{x} D_{y}\left(R^{\alpha} u\right), \\
& \zeta_{y}^{\alpha}=D_{y} R^{\alpha}\left[\eta-\xi^{0} u_{t}-\xi^{1} u_{x}-\xi^{2} u_{y}\right]+\xi^{0} D_{y} D_{t}\left(R^{\alpha} u\right)+\xi^{1} D_{y} D_{x}\left(R^{\alpha} u\right)+\xi^{2} D_{y}^{2}\left(R^{\alpha} u\right), \\
& \zeta_{x x}^{\alpha}=D_{x}^{2} R^{\alpha}\left[\eta-\xi^{0} u_{t}-\xi^{1} u_{x}-\xi^{2} u_{y}\right]+\xi^{0} D_{x}^{2} D_{t}\left(R^{\alpha} u\right)+\xi^{1} D_{x}^{3}\left(R^{\alpha} u\right)+\xi^{2} D_{x}^{2} D_{y}\left(R^{\alpha} u\right), \\
& \zeta_{y y}^{\alpha}=D_{y}^{2} R^{\alpha}\left[\eta-\xi^{0} u_{t}-\xi^{1} u_{x}-\xi^{2} u_{y}\right]+\xi^{0} D_{y}^{2} D_{t}\left(R^{\alpha} u\right)+\xi^{1} D_{y}^{2} D_{x}\left(R^{\alpha} u\right)+\xi^{2} D_{y}^{3}\left(R^{\alpha} u\right) .
\end{aligned}
$$

Acting by the generator (20) on the Equation (19), we obtain the determining equation:

$$
\begin{aligned}
& \zeta_{t}=k(u)\left(\zeta_{x x}^{\alpha}+\zeta_{y y}^{\alpha}\right)+k^{\prime}(u) \eta\left[D_{x}^{2}\left(R^{\alpha} u\right)+D_{y}^{2}\left(R^{\alpha} u\right)\right] \\
& +k^{\prime}(u)\left[\zeta_{x} D_{x}\left(R^{\alpha} u\right)+\zeta_{y} D_{y}\left(R^{\alpha} u\right)+u_{x} \zeta_{x}^{\alpha}+u_{y} \zeta_{y}^{\alpha}\right]+k^{\prime \prime}(u) \eta\left[u_{x} D_{x}\left(R^{\alpha} u\right)+u_{y} D_{y}\left(R^{\alpha} u\right)\right] .
\end{aligned}
$$

We substitute the prolongation formulae (21), (22) into Equation (23) and replace $D_{y}^{2}\left(R^{\alpha} u\right)$ in view of Equation (19) as

$$
D_{y}^{2}\left(R^{\alpha} u\right)=k^{-1}(u)\left[u_{t}-k(u) D_{x}^{2}\left(R^{\alpha} u\right)-k^{\prime}(u) u_{x} D_{x}\left(R^{\alpha} u\right)-k^{\prime}(u) u_{y} D_{y}\left(R^{\alpha} u\right)\right] .
$$

Then, we use the generalized Leibniz rule (14) and its consequences (15). Taking into account that $R^{\alpha} u=I_{2-\alpha}^{0,0} u$, we represent all terms with $R^{\alpha} u$ and their derivatives via integral operators (12). By applying the recurrence relation (13), we rewrite all integrals $I_{k-\alpha}^{m, n} u, I_{k-\alpha}^{m, n} u_{t}$ in terms of $I_{8-\alpha}^{m, n} u$ and $I_{8-\alpha}^{m, n} u_{t}$. As a result, we obtain the determining equation in which $u_{t}, u_{x}, u_{y}, D_{x}^{2}\left(R^{\alpha} u\right), I_{8-\alpha}^{m, n} u, I_{8-\alpha}^{m, n} u_{t}$ can be considered as independent variables. Splitting the obtained equation by all these variables, we get an infinite system of integer-order partial differential equations and one fractional differential equation. We do not write here the whole obtained system due to its large size, but we present a particular result of the splitting which leads to the classifying relation.

By equating to zero the coefficients for $u_{t}$, we obtain

$$
\xi_{x}^{0}=0, \quad \xi_{y}^{0}=0, \quad \xi_{t}^{0}=(2-\alpha) \xi_{y}^{2}-\frac{k^{\prime}(u)}{k(u)} \eta
$$

Thus, $\xi^{0}=\xi^{0}(t)$. Since $\xi^{0}$ and $\xi^{2}$ do not depend on $u$, and $\eta$ is a linear function with respect to $u$, from the last equation in (24), we obtain the classifying relation for $k(u) \neq$ const:

$$
\left(\frac{1}{K(u)}\right)^{\prime \prime}=0, \quad K(u)=\frac{k^{\prime}(u)}{k(u)}, \quad k^{\prime}(u) \neq 0 .
$$


This relation is exactly the same as for the integer-order nonlinear heat equation [11] and as for the time-fractional nonlinear diffusion equation [18]. From this classifying relation, in view of the equivalence transformations (18), we obtain that the following cases should be distinguished:

1. $k(u)$ is an arbitrary function;

2. $k(u)=e^{u}$;

3. $k(u)=(u+A)^{\sigma}, \sigma \neq 0, A=$ const;

4. $k(u)=1$.

The subsequent analysis shows that there is no extension of the symmetry group for $k(u)=e^{u}$, and for $k(u)=(u+A)^{\sigma}$ the symmetry group is extended only with $A=0$. The main reason for these results is that the integral $R^{\alpha}(1)$ diverges.

The final results of group classification for Equation (16) are summarized in the following theorem.

Theorem 4. The nonlinear Equation (16) with an arbitrary functions $k(u)$ has a five-parameter Lie point symmetry group spanned by the generators

$$
\begin{gathered}
X_{1}=\frac{\partial}{\partial t}, \quad X_{2}=\frac{\partial}{\partial x}, \quad X_{3}=\frac{\partial}{\partial y}, \\
X_{4}=(2-\alpha) t \frac{\partial}{\partial t}+x \frac{\partial}{\partial x}+y \frac{\partial}{\partial y}, \quad X_{5}=y \frac{\partial}{\partial x}-x \frac{\partial}{\partial y} .
\end{gathered}
$$

This symmetry group is extended only for $k(u)=u^{\sigma}(\sigma \in R)$ :

$$
X_{6}=\sigma t \frac{\partial}{\partial t}-u \frac{\partial}{\partial u}
$$

for arbitrary $\sigma$;

$$
X_{\infty}^{1}=g(t, x, y) \frac{\partial}{\partial u}
$$

for $\sigma=0$ (linear case), where $g(t, x, y)$ is an arbitrary solution of the linear equation $g_{t}=\Delta R^{\alpha} g$;

$$
X_{\infty}^{2}=A \frac{\partial}{\partial x}+B \frac{\partial}{\partial y}-2 A_{x} u \frac{\partial}{\partial u}
$$

for $\sigma=-1$, where $A(x, y), B(x, y)$ are the arbitrary solutions of the system

$$
A_{x}=B_{y}, \quad A_{y}=-B_{x} .
$$

The symmetries given in this theorem can be used for finding group invariant solutions and conservation laws of the equation in question. We do not present here invariant solutions because their construction is a problem for future research. Now, we focus on the problem of conservation laws finding.

\section{Nonlinear Self-Adjointness}

In this section, we extend the basic notions of the concept of nonlinear self-adjointness to FDEs with the Riesz potential and prove that Equation (16) is nonlinearly self-adjoint. We will assume that $n>1$ and $u$ is not a radial function since otherwise known results $[15,23,27]$ for fractional differential equations with the Riemann-Liouville fractional derivatives can be used (see Remark 1).

Let us consider the function

$$
F=F\left(x^{0}, x, u, u_{(1)}, \ldots, u_{(k)},\left(R^{\alpha} u\right)_{(1)}, \ldots,\left(R^{\alpha} u\right)_{(m)}\right),
$$


where $u=u\left(x^{0}, x\right), x^{0} \in[0, T]$ is the time variable and $x=\left(x^{1}, \ldots, x^{n}\right) \in R^{n}$ is the vector of spatial variables. In (28), we use the following notation of differential algebra (see, e.g., [13]):

$$
v_{(1)}=\left\{v_{i_{1}}\right\}, \ldots, v_{(k)}=\left\{v_{i_{1} \ldots i_{k}}\right\} ; \quad v_{i_{1}}=D_{i_{1}}(v), \ldots, v_{i_{1} \ldots i_{k}}=D_{i_{k}}\left(v_{i_{1} \ldots i_{k-1}}\right)=D_{i_{k}} \ldots D_{i_{1}}(v)
$$

with $i_{1}, \ldots, i_{k}=0, \ldots, n$. Here, $D_{i}$ denotes the operator of total differentiation with respect to $x^{i}$.

First of all, we obtain an explicit representation for the variational derivative of the function $F$ in (28). The corresponding variational integral has the form

$$
S[u]=\int_{0}^{T} \int_{R^{n}} F\left(x^{0}, x, u, u_{(1)}, \ldots, u_{(k)},\left(R^{\alpha} u\right)_{(1)}, \ldots,\left(R^{\alpha} u\right)_{(m)}\right) d x d x^{0} .
$$

The first variation of the functional $S[u]$ can be found as

$$
\delta S[u]=\left.\left(\frac{\partial}{\partial \varepsilon} S[u+\varepsilon \delta u]\right)\right|_{\varepsilon=0},
$$

where $\delta u$ is a variation of the function $u$. As usual in calculus of variations, we will assume that $\delta u, R^{\alpha}(\delta u)$ and all their derivatives with respect to all variables $x^{i}(i=0,1, \ldots, n)$ are equal to zero for $|x| \rightarrow \infty, x^{0}=0$ and $x^{0}=T$. Since the Riesz potential and any differential operator are linear, after simple algebra, we obtain

$$
\delta S[u]=\int_{0}^{T} \int_{R^{n}}\left[\frac{\partial F}{\partial u} \delta u+\sum_{s=1}^{k} \frac{\partial F}{\partial u_{i_{1} \ldots i_{s}}} D_{i_{s}} \ldots D_{i_{1}}(\delta u)+\sum_{r=1}^{m} \frac{\partial F}{\partial\left(R^{\alpha} u\right)_{j_{1} \ldots j_{r}}} D_{j_{r}} \ldots D_{j_{1}}\left(R^{\alpha} \delta u\right)\right] d x d x^{0} .
$$

The multiple integration by parts yields

$$
\begin{aligned}
\delta S[u]=\int_{0}^{T} \int_{R^{n}}\left[\frac{\partial F}{\partial u} \delta u+\sum_{s=1}^{k}(-1)^{s} D_{i_{1}} \ldots D_{i_{s}}\right. & \left(\frac{\partial F}{\partial u_{i_{1} \ldots i_{s}}}\right) \delta u \\
& \left.+\sum_{r=1}^{m}(-1)^{r} D_{j_{1}} \ldots D_{j_{r}}\left(\frac{\partial F}{\partial\left(R^{\alpha} u\right)_{j_{1} \ldots j_{r}}}\right)\left(R^{\alpha} \delta u\right)\right] d x d x^{0} .
\end{aligned}
$$

Let $f(x)$ and $g(x)$ be such functions that $R^{\alpha} f, R^{\alpha} g$, and the integral $\int_{R^{n}} f R^{\alpha} g d x$ exist. Then, it is easy to prove that

$$
\int_{R^{n}} f R^{\alpha} g d x=\int_{R^{n}} g R^{\alpha} f d x
$$

Using this property of the Riesz potential, we get

$$
\begin{aligned}
& \delta S[u]=\int_{0}^{T} \int_{R^{n}}\left[\frac{\partial F}{\partial u}+\sum_{s=1}^{k}(-1)^{s} D_{i_{1}} \ldots D_{i_{s}}\left(\frac{\partial F}{\partial u_{i_{1} \ldots i_{s}}}\right)\right. \\
& \left.+\sum_{r=1}^{m}(-1)^{r} R^{\alpha} D_{j_{1}} \ldots D_{j_{r}}\left(\frac{\partial F}{\partial\left(R^{\alpha} u\right)_{j_{1} \ldots j_{r}}}\right)\right](\delta u) d x d x^{0} .
\end{aligned}
$$

If, for any $\delta u$ we have $\delta S[u]=0$, then the function $u$ extremize the functional $S[u]$. It follows from (30) that this function can be found as a solution of the fractional generalization of the Euler-Lagrange equation

$$
\frac{\delta F}{\delta u}=0
$$

where

$$
\frac{\delta}{\delta u}=\frac{\partial}{\partial u}+\sum_{s=1}^{k}(-1)^{s} D_{i_{1}} \ldots D_{i_{s}}\left(\frac{\partial}{\partial u_{i_{1} \ldots i_{s}}}\right)+\sum_{r=1}^{m}(-1)^{r} R^{\alpha} D_{j_{1}} \ldots D_{j_{r}}\left(\frac{\partial}{\partial\left(R^{\alpha} u\right)_{j_{1} \ldots j_{r}}}\right)
$$


is the Euler-Lagrange operator (operator of variational derivative) for the integral (29).

Now, let us consider a fractional differential equation

$$
F\left(x^{0}, x, u, u_{(1)}, \ldots, u_{(k)},\left(R^{\alpha} u\right)_{(1)}, \ldots,\left(R^{\alpha} u\right)_{(m)}\right)=0 .
$$

Following Ibragimov $[25,26]$, we introduce the formal Lagrangian

$$
\mathcal{L}=v\left(x^{0}, x\right) F\left(x^{0}, x, u, u_{(1)}, \ldots, u_{(k)},\left(R^{\alpha} u\right)_{(1)}, \ldots,\left(R^{\alpha} u\right)_{(m)}\right)
$$

where $v\left(x^{0}, x\right)$ is a new dependent variable, and define the function

$$
F^{*}\left(x^{0}, x, u, v, u_{(1)}, v_{(1)}, \ldots, u_{(k)}, v_{(k)},\left(R^{\alpha} u\right)_{(1)}, R^{\alpha}\left(v_{(1)}\right), \ldots,\left(R^{\alpha} u\right)_{(m)}, R^{\alpha}\left(v_{(m)}\right)\right)=\frac{\delta(v F)}{\delta u} .
$$

Then,

$$
F^{*}\left(x^{0}, x, u, v, u_{(1)}, v_{(1)}, \ldots, u_{(k)}, v_{(k)},\left(R^{\alpha} u\right)_{(1)}, R^{\alpha}\left(v_{(1)}\right), \ldots,\left(R^{\alpha} u\right)_{(m)}, R^{\alpha}\left(v_{(m)}\right)\right)=0
$$

is the adjoint equation to Equation (32).

Similarly to [25,26], Equation (32) will be called nonlinearly self-adjoint if the adjoint Equation (33) will be satisfied for all solutions $u\left(x^{0}, x\right)$ of Equation (32) upon a substitution

$$
v=\varphi\left(x^{0}, x, u\right), \quad \varphi \neq 0 .
$$

Theorem 5. The nonlinear Equation (16) is nonlinearly self-adjoint.

Proof. It is easy to see that Equation (16) is a particular case of the equation

$$
F\left(t, x, y, u, u_{t}, u_{x}, u_{y}, D_{x}\left(R^{\alpha} u\right), D_{y}\left(R^{\alpha} u\right), D_{x}^{2}\left(R^{\alpha} u\right), D_{y}^{2}\left(R^{\alpha} u\right)\right)=0 .
$$

Then, the Euler-Lagrange operator (31) has the form

$$
\begin{aligned}
\frac{\delta}{\delta u}=\frac{\partial}{\partial u}-D_{t} \frac{\partial}{\partial u_{t}}-D_{x} \frac{\partial}{\partial u_{x}}-D_{y} \frac{\partial}{\partial u_{y}}-R^{\alpha} D_{x} \frac{\partial}{\partial\left(D_{x} R^{\alpha} u\right)}- & R^{\alpha} D_{y} \frac{\partial}{\partial\left(D_{y} R^{\alpha} u\right)} \\
& +R^{\alpha} D_{x}^{2} \frac{\partial}{\partial\left(D_{x}^{2} R^{\alpha} u\right)}+R^{\alpha} D_{y}^{2} \frac{\partial}{\partial\left(D_{y}^{2} R^{\alpha} u\right)}
\end{aligned}
$$

The corresponding adjoint equation

$$
\frac{\delta(v F)}{\delta u}=0
$$

can be written as

$$
-v_{t}+k^{\prime}(u)\left[v_{x} D_{x}\left(R^{\alpha} u\right)+v_{y} D_{y}\left(R^{\alpha} u\right)\right]-R^{\alpha}\left[D_{x}\left(k v_{x}\right)+D_{y}\left(k v_{y}\right)\right]=0 .
$$

The substitution (34) takes the form $v=\varphi(t, x, y, u)$. Then, Equation (35) is transformed into

$$
\begin{aligned}
-\varphi_{t}-\varphi_{u} u_{t}+k^{\prime}(u)\left[\left(\varphi_{x}+\varphi_{u} u_{x}\right) D_{x}\left(R^{\alpha} u\right)+\right. & \left.\left(\varphi_{y}+\varphi_{u} u_{y}\right) D_{y}\left(R^{\alpha} u\right)\right] \\
& -R^{\alpha}\left[D_{x}\left(k\left(\varphi_{x}+\varphi_{u} u_{x}\right)\right)+D_{y}\left(k\left(\varphi_{y}+\varphi_{u} u_{y}\right)\right)\right]=0 .
\end{aligned}
$$

It is evident that this equation holds identically for $\varphi=c(c=$ const $)$. Therefore, Equation (16) is nonlinearly self-adjoint. 


\section{Conservation Laws}

We will use the classical definition of a conservation law (see, e.g., [11]) since it is suitable for most applications. In such a way, the conservation law for Equation (32) can be written as

$$
D_{i}\left(C^{i}\right)=0, \quad i=0,1, \ldots, n,
$$

where $C=\left(C^{0}, \ldots, C^{n}\right)$ is the so-called conserved vector. Other approaches for fractional differential equations can be found in [32-34].

In [22], it is shown that the components of a conserved vector can be found as

$$
C^{i}=\mathcal{N}^{i} \mathcal{L},
$$

where $\mathcal{L}$ is a classical or formal Lagrangian, and $\mathcal{N}^{i}(i=0,1, \ldots, n)$ are the so-called Noether operators. These operators are defined by the fundamental operator identity

$$
\tilde{X}+D_{i}\left(\xi^{i}\right) \mathcal{I}=W \frac{\delta}{\delta u}+D_{i}\left(\mathcal{N}^{i}\right)
$$

where $\tilde{X}$ is an appropriate prolongation of the Lie point group generator to all dependent variables in the considered equation, $\mathcal{I}$ is the identity operator, $W=\eta-\xi^{i} u_{i}$, and $\frac{\delta}{\delta u}$ is the Euler-Lagrange operator.

For Equation (32), the prolonged generator $\tilde{X}$ can be written in the form

$$
\tilde{X}=\xi^{i} \frac{\partial}{\partial x^{i}}+\eta \frac{\partial}{\partial u}+\sum_{s=1}^{k} \zeta_{i_{1} \ldots i_{s}} \frac{\partial}{\partial u_{i_{1} \ldots i_{s}}}+\sum_{r=1}^{m} \zeta_{j_{1} \ldots j_{r}}^{\alpha} \frac{\partial}{\partial\left(R^{\alpha} u\right)_{j_{1} \ldots j_{r}}},
$$

where $i_{1}, \ldots, i_{k}=0,1, \ldots, n$ and $j_{1}, \ldots, j_{m}=0,1, \ldots, n$. The functions $\zeta_{i_{1} \ldots i_{s}}$ and $\zeta_{j_{1} \ldots j_{r}}^{\alpha}$ are given by the prolongation formulae

$$
\begin{gathered}
\zeta_{i_{1} \ldots i_{s}}=D_{i_{s}} \ldots D_{i_{1}}(W)+\xi^{l} D_{l}\left(u_{i_{1} \ldots i_{s}}\right), \\
\zeta_{j_{1} \ldots j_{r}}^{\alpha}=D_{j_{r}} \ldots D_{j_{1}}\left(R^{\alpha} W\right)+\xi^{l} D_{l}\left(\left(R^{\alpha} u\right)_{j_{1} \ldots j_{r}}\right) .
\end{gathered}
$$

By applying (38) to the function $F$ defined in (28) and using (31), we get

$$
\begin{array}{r}
D_{i}\left(\mathcal{N}^{i} F\right)=\tilde{X} F+D_{i}\left(\xi^{i}\right) F-W \frac{\delta F}{\delta u}=\xi^{i} \frac{\partial F}{\partial x^{i}}+\eta \frac{\partial F}{\partial u}+\sum_{s=1}^{k}\left[D_{i_{s}} \ldots D_{i_{1}}(W)+\xi^{l} D_{l}\left(u_{i_{1} \ldots i_{s}}\right)\right] \frac{\partial F}{\partial u_{i_{1} \ldots i_{s}}} \\
+\sum_{r=1}^{m}\left[D_{j_{r}} \ldots D_{j_{1}}\left(R^{\alpha} W\right)+\xi^{l} D_{l}\left(\left(R^{\alpha} u\right)_{j_{1} \ldots j_{r}}\right)\right] \frac{\partial F}{\partial\left(R^{\alpha} u\right)_{j_{1} \ldots j_{r}}}+D_{i}\left(\xi^{i}\right) F-\left(\eta-\xi^{i} u_{i}\right) \frac{\partial F}{\partial u} \\
-W \sum_{s=1}^{k}(-1)^{s} D_{i_{1}} \ldots D_{i_{s}}\left(\frac{\partial F}{\partial u_{i_{1} \ldots i_{s}}}\right)-W \sum_{r=1}^{m}(-1)^{r} R^{\alpha} D_{j_{1}} \ldots D_{j_{r}}\left(\frac{\partial F}{\partial\left(R^{\alpha} u\right)_{j_{1} \ldots j_{r}}}\right) .
\end{array}
$$

Note that

$$
D_{i}(F)=\frac{\partial F}{\partial x^{i}}+u_{i} \frac{\partial F}{\partial u}+\sum_{s=1}^{k} u_{i_{1} \ldots i_{s} i} \frac{\partial F}{\partial u_{i_{1} \ldots i_{s}}}+\sum_{r=1}^{m}\left(R^{\alpha} u\right)_{j_{1} \ldots j_{r} i} \frac{\partial F}{\partial\left(R^{\alpha} u\right)_{j_{1} \ldots j_{r}}}
$$

and

$$
\xi^{i} D_{i}(F)+D_{i}\left(\xi^{i}\right) F=D_{i}\left(\xi^{i} F\right)
$$


Then, we have

$$
\begin{aligned}
& D_{i}\left(\mathcal{N}^{i} F\right)=D_{i}\left(\xi^{i} F\right)+\sum_{s=1}^{k}\left[D_{i_{s}} \ldots D_{i_{1}}(W) \cdot \frac{\partial F}{\partial u_{i_{1} \ldots i_{s}}}-(-1)^{s} W \cdot D_{i_{1}} \ldots D_{i_{s}}\left(\frac{\partial F}{\partial u_{i_{1} \ldots i_{s}}}\right)\right] \\
& +\sum_{r=1}^{m}\left[D_{j_{r}} \ldots D_{j_{1}}\left(R^{\alpha} W\right) \cdot \frac{\partial F}{\partial\left(R^{\alpha} u\right)_{j_{1} \ldots j_{r}}}-(-1)^{r} W \cdot R^{\alpha} D_{j_{1}} \ldots D_{j_{r}}\left(\frac{\partial F}{\partial\left(R^{\alpha} u\right)_{j_{1} \ldots j_{r}}}\right)\right] .
\end{aligned}
$$

For the first sum on the right-hand side of Equation (40), we can use the known representation $[22,26]$

$$
\begin{aligned}
& \sum_{s=1}^{k}\left[D_{i_{s}} \ldots D_{i_{1}}(W) \cdot \frac{\partial F}{\partial u_{i_{1} \ldots i_{s}}}-(-1)^{s} W \cdot D_{i_{1}} \ldots D_{i_{s}}\left(\frac{\partial F}{\partial u_{i_{1} \ldots i_{s}}}\right)\right] \\
& =D_{i}\left[W\left(\frac{\partial F}{\partial u_{i}}+\sum_{s=1}^{k-1}(-1)^{s} D_{i_{1}} \ldots D_{i_{s}} \frac{\partial F}{\partial u_{i i_{1} \ldots i_{s}}}\right)\right. \\
& \left.+\sum_{r=1}^{k-1} D_{l_{1}} \ldots D_{l_{r}}(W)\left(\frac{\partial F}{\partial u_{i l_{1} \ldots l_{r}}}+\sum_{s=1}^{k-1-r}(-1)^{s} D_{i_{1}} \ldots D_{i_{s}} \frac{\partial F}{\partial u_{i l_{1} \ldots l_{r} i_{1} \ldots i_{s}}}\right)\right] .
\end{aligned}
$$

The second sum on the right-hand side of Equation (40) contains the Riesz operator and therefore a new approach is needed. In [23,27], it is shown that, for equations with the Riemann-Liouville and Caputo fractional derivatives, the corresponding Noether operators can be written in an explicit form by using special integral operators. Similar but more complex operators can be introduced for the equations with the Riesz potential.

We define the operator $J_{(i)}^{\alpha}(\alpha \in(0,1))$ acting on an ordered pair of functions $\left\{f\left(x^{0}, x\right), g\left(x^{0}, x\right)\right\}$ $\left(x^{0} \in[0, T], x \in R^{n}\right)$ by

$$
\begin{aligned}
& J_{(i)}^{\alpha}\left\{f\left(x^{0}, x\right), g\left(x^{0}, x\right)\right\}=\frac{1}{\gamma_{n}(\alpha)} \int_{-\infty}^{x^{i}} \int_{R^{n}} \frac{\left.f\left(x^{0}, x\right)\right|_{x^{i}=\rho} g\left(x^{0}, \mu\right)}{\left.\left(|x-\mu|^{n-\alpha}\right)\right|_{x^{i}=\rho}} d \mu d \rho \\
& +\frac{1}{\gamma_{n}(\alpha)} \int_{x^{i}}^{\infty} \int_{R^{n}} \frac{\left.f\left(x^{0}, \mu\right) g\left(x^{0}, x\right)\right|_{x^{i}=\rho}}{\left.\left(|x-\mu|^{n-\alpha}\right)\right|_{x^{i}=\rho}} d \mu d \rho
\end{aligned}
$$

for $i=1, \ldots, n$, and by

$$
J_{(0)}^{\alpha}\left\{f\left(x^{0}, x\right), g\left(x^{0}, x\right)\right\}=\frac{1}{\gamma_{n}(\alpha)} \int_{0}^{x^{0}} \int_{R^{n}} \frac{f(\rho, x) g\left(x^{0}, \mu\right)}{|x-\mu|^{n-\alpha}} d \mu d \rho+\frac{1}{\gamma_{n}(\alpha)} \int_{x^{0}}^{T} \int_{R^{n}} \frac{f\left(x^{0}, \mu\right) g(\rho, x)}{|x-\mu|^{n-\alpha}} d \mu d \rho,
$$

for $i=0$. It is easy to show by the direct computation that the following equality holds:

$$
D_{i}\left(J_{(i)}^{\alpha}\{f(x), g(x)\}\right)=f R^{\alpha} g-g R^{\alpha} f
$$

(here $(i)$ means that there is no summation with respect to $i$ ). In Equation (44), we assume that $f\left(x^{0}, x\right)$ and $g\left(x^{0}, x\right)$ belong to an appropriate class of functions such that all integrals exist. For completeness, we will assume that

$$
J_{(i)}^{0}\left\{f\left(x^{0}, x\right), g\left(x^{0}, x\right)\right\}=0, \quad i=0,1, \ldots, n .
$$

By using (42) and (44), we obtain

$$
D_{i}\left(R^{\alpha} W\right) \cdot \frac{\partial F}{\partial\left(R^{\alpha} u\right)_{i}}+W \cdot R^{\alpha} D_{i}\left(\frac{\partial F}{\partial\left(R^{\alpha} u\right)_{i}}\right)=D_{i}\left(R^{\alpha} W \cdot \frac{\partial F}{\partial\left(R^{\alpha} u\right)_{i}}+J_{(i)}^{\alpha}\left\{W, D_{i}\left(\frac{\partial F}{\partial\left(R^{\alpha} u\right)_{i}}\right)\right\}\right) .
$$


By using (41), we can rewrite the second sum on the right-hand side of Equation (40) in the form

$$
\begin{aligned}
& \sum_{r=1}^{m}\left[D_{j_{r}} \ldots D_{j_{1}}\left(R^{\alpha} W\right) \cdot \frac{\partial F}{\partial\left(R^{\alpha} u\right)_{j_{1} \ldots j_{r}}}-(-1)^{r} W \cdot R^{\alpha} D_{j_{1}} \ldots D_{j_{r}}\left(\frac{\partial F}{\partial\left(R^{\alpha} u\right)_{j_{1} \ldots j_{r}}}\right)\right] \\
& =D_{i}\left[\left(R^{\alpha} W\right)\left(\frac{\partial F}{\partial\left(R^{\alpha} u\right)_{i}}+\sum_{r=1}^{m-1}(-1)^{r} D_{j_{1}} \ldots D_{j_{r}} \frac{\partial F}{\partial\left(R^{\alpha} u\right)_{i j_{1} \ldots j_{r}}}\right)\right. \\
& \left.+\sum_{s=1}^{m-1} D_{l_{1} \ldots D_{l_{s}}}\left(R^{\alpha} W\right)\left(\frac{\partial F}{\partial\left(R^{\alpha} u\right)_{i l_{1} \ldots l_{s}}}+\sum_{r=1}^{m-1-s}(-1)^{r} D_{j_{1}} \ldots D_{j_{r}} \frac{\partial F}{\partial\left(R^{\alpha} u\right)_{i l_{1} \ldots l_{s} j_{1} \ldots j_{r}}}\right)\right] \\
& +\sum_{r=1}^{m}(-1)^{r} R^{\alpha} W \cdot D_{j_{1}} \ldots D_{j_{r}}\left(\frac{\partial F}{\partial\left(R^{\alpha} u\right)_{j_{1} \ldots j_{r}}}\right)-\sum_{r=1}^{m}(-1)^{r} W \cdot R^{\alpha} D_{j_{1}} \ldots D_{j_{r}}\left(\frac{\partial F}{\partial\left(R^{\alpha} u\right)_{j_{1} \ldots j_{r}}}\right) .
\end{aligned}
$$

In view of Equality (44), the two last terms in this expression can be written as

$$
\begin{aligned}
\sum_{r=1}^{m}(-1)^{r} R^{\alpha} W \cdot D_{j_{1}} \ldots D_{j_{r}}\left(\frac{\partial F}{\partial\left(R^{\alpha} u\right)_{j_{1} \ldots j_{r}}}\right) & -\sum_{r=1}^{m}(-1)^{r} W \cdot R^{\alpha} D_{j_{1}} \ldots D_{j_{r}}\left(\frac{\partial F}{\partial\left(R^{\alpha} u\right)_{j_{1} \ldots j_{r}}}\right) \\
& =-D_{i}\left[\sum_{r=1}^{m}(-1)^{r} J_{(i)}^{\alpha}\left\{W, D_{j_{1}} \ldots D_{j_{r}}\left(\frac{\partial F}{\partial\left(R^{\alpha} u\right)_{j_{1} \ldots j_{r}}}\right)\right\}\right]
\end{aligned}
$$

By substitution of all obtained expressions for sums into the right-hand side of the Equality (40), we derive the following explicit representations for the Noether operators:

$$
\begin{aligned}
\mathcal{N}^{i} F=\xi^{i} F+W & \left(\frac{\partial F}{\partial u_{i}}+\sum_{s=1}^{k-1}(-1)^{s} D_{i_{1}} \ldots D_{i_{s}} \frac{\partial F}{\partial u_{i i_{1} \ldots i_{s}}}\right) \\
+ & \sum_{r=1}^{k-1} D_{l_{1}} \ldots D_{l_{r}}(W)\left(\frac{\partial F}{\partial u_{i l_{1} \ldots l_{r}}}+\sum_{s=1}^{k-1-r}(-1)^{s} D_{i_{1}} \ldots D_{i_{s}} \frac{\partial F}{\partial u_{i l_{1} \ldots l_{r} i_{1} \ldots i_{s}}}\right) \\
& +\left(R^{\alpha} W\right)\left(\frac{\partial F}{\partial\left(R^{\alpha} u\right)_{i}}+\sum_{r=1}^{m-1}(-1)^{r} D_{j_{1}} \ldots D_{j_{r}} \frac{\partial F}{\partial\left(R^{\alpha} u\right)_{i j_{1} \ldots j_{r}}}\right) \\
+ & \sum_{s=1}^{m-1} D_{l_{1}} \ldots D_{l_{s}}\left(R^{\alpha} W\right)\left(\frac{\partial F}{\partial\left(R^{\alpha} u\right)_{i l_{1} \ldots l_{s}}}+\sum_{r=1}^{m-1-s}(-1)^{r} D_{j_{1}} \ldots D_{j_{r}} \frac{\partial F}{\partial\left(R^{\alpha} u\right)_{i l_{1} \ldots l_{s} j_{1} \ldots j_{r}}}\right) \\
& -\sum_{r=1}^{m}(-1)^{r} J_{(i)}^{\alpha}\left\{W, D_{j_{1}} \ldots D_{j_{r}}\left(\frac{\partial F}{\partial\left(R^{\alpha} u\right)_{j_{1} \ldots j_{r}}}\right)\right\} .
\end{aligned}
$$

Thus, the conservation laws for any fractional differential equation of the form (32) can be obtained by (37) with (46). To illustrate this approach, we construct conservation laws for the nonlinear fractional diffusion Equation (16).

The conservation law (36) for Equation (16) takes the form

$$
D_{t}\left(C^{t}\right)+D_{x}\left(C^{x}\right)+D_{y}\left(C^{y}\right)=0 .
$$

In the previous section, it is proved that Equation (16) is nonlinearly self-adjoint and the substitution (34) is $v=c=$ const. Without loss of generality, we can set $c=1$ because any conservation law is linear with respect to conserved vector's components. Then, the formal Lagrangian coincides with the equation:

$$
\mathcal{L}=F \equiv u_{t}-k(u)\left[D_{x}^{2}\left(R^{\alpha} u\right)+D_{y}^{2}\left(R^{\alpha} u\right)\right]-k^{\prime}(u) u_{x} D_{x}\left(R^{\alpha} u\right)-k^{\prime}(u) u_{y} D_{y}\left(R^{\alpha} u\right) .
$$


For this function, the Noether operators (46) takes a more simple form:

$$
\begin{aligned}
& \mathcal{N}^{t} F=\xi^{0} F+W \frac{\partial F}{\partial u_{t}}, \\
& \begin{aligned}
\mathcal{N}^{x} F=\xi^{1} F+W \frac{\partial F}{\partial u_{x}}+R^{\alpha} W & {\left[\frac{\partial F}{\partial\left(D_{x} R^{\alpha} u\right)}-D_{x}\left(\frac{\partial F}{\partial\left(D_{x}^{2} R^{\alpha} u\right)}\right)\right]+D_{x}\left(R^{\alpha} W\right) \frac{\partial F}{\partial\left(D_{x}^{2} R^{\alpha} u\right)} } \\
& +J_{(1)}^{\alpha}\left\{W, D_{x}\left(\frac{\partial F}{\partial\left(D_{x} R^{\alpha} u\right)}\right)\right\}-J_{(1)}^{\alpha}\left\{W, D_{x}^{2}\left(\frac{\partial F}{\partial\left(D_{x}^{2} R^{\alpha} u\right)}\right)\right\}, \\
\mathcal{N}^{y} F=\xi^{2} F+W \frac{\partial F}{\partial u_{y}}+R^{\alpha} W & {\left[\frac{\partial F}{\partial\left(D_{y} R^{\alpha} u\right)}-D_{y}\left(\frac{\partial F}{\partial\left(D_{y}^{2} R^{\alpha} u\right)}\right)\right]+D_{y}\left(R^{\alpha} W\right) \frac{\partial F}{\partial\left(D_{y}^{2} R^{\alpha} u\right)} } \\
& +J_{(2)}^{\alpha}\left\{W, D_{y}\left(\frac{\partial F}{\partial\left(D_{y} R^{\alpha} u\right)}\right)\right\}-J_{(2)}^{\alpha}\left\{W, D_{y}^{2}\left(\frac{\partial F}{\partial\left(D_{y}^{2} R^{\alpha} u\right)}\right)\right\} .
\end{aligned}
\end{aligned}
$$

Substituting (47) and (48) into (37), in view of the equation $F=0$, we obtain

$$
\begin{aligned}
& C^{t}=W, \\
& C^{x}=-\left[k^{\prime}(u) W D_{x}\left(R^{\alpha} u\right)+k(u) D_{x}\left(R^{\alpha} W\right)\right], \\
& C^{y}=-\left[k^{\prime}(u) W D_{y}\left(R^{\alpha} u\right)+k(u) D_{y}\left(R^{\alpha} W\right)\right] .
\end{aligned}
$$

Here, we use the linearity of operators $J_{(i)}^{\alpha}$ :

$$
\begin{aligned}
J_{(1)}^{\alpha}\left\{W, D_{x}\left(\frac{\partial F}{\partial\left(D_{x} R^{\alpha} u\right)}\right)\right\} & -J_{(1)}^{\alpha}\left\{W, D_{x}^{2}\left(\frac{\partial F}{\partial\left(D_{x}^{2} R^{\alpha} u\right)}\right)\right\} \\
= & J_{(1)}^{\alpha}\left\{W, D_{x}\left(\frac{\partial F}{\partial\left(D_{x} R^{\alpha} u\right)}\right)-D_{x}^{2}\left(\frac{\partial F}{\partial\left(D_{x}^{2} R^{\alpha} u\right)}\right)\right\} \\
& =J_{(1)}^{\alpha}\left\{W, D_{x}\left(-k^{\prime}(u) u_{x}\right)-D_{x}^{2}(-k(u))\right\}=J_{(1)}^{\alpha}\{W, 0\}=0 .
\end{aligned}
$$

The similar equality holds for $J_{(2)}^{\alpha}$.

Let us consider the case when $k(u)$ is an arbitrary function. Then, Equation (16) admits five-parameter Lie point symmetry group with the basis (25). For these basis operators, we have

$$
W_{1}=-u_{t}, \quad W_{2}=-u_{x}, \quad W_{3}=-u_{y}, \quad W_{4}=(\alpha-2) t u_{t}-x u_{x}-y u_{y}, \quad W_{5}=x u_{y}-y u_{x} .
$$

The conservation law corresponding to $W_{1}$ is trivial. Indeed, in view of the Equation (16), we have

$$
\begin{aligned}
& C_{1}^{t}=-u_{t}=-D_{x}\left(k(u) D_{x} R^{\alpha} u\right)-D_{y}\left(k(u) D_{y} R^{\alpha} u\right), \\
& C_{1}^{x}=k^{\prime}(u) u_{t} D_{x}\left(R^{\alpha} u\right)+k(u) D_{x}\left(R^{\alpha} u_{t}\right), \\
& C_{1}^{y}=k^{\prime}(u) u_{t} D_{y}\left(R^{\alpha} u\right)+k(u) D_{y}\left(R^{\alpha} u_{t}\right) .
\end{aligned}
$$

Then, the conservation law reads

$$
\begin{aligned}
D_{t}\left(C_{1}^{t}\right)+D_{x}\left(C_{1}^{x}\right)+D_{y}\left(C_{1}^{y}\right)=D_{t}[ & \left.-D_{x}\left(k(u) D_{x} R^{\alpha} u\right)-D_{y}\left(k(u) D_{y} R^{\alpha} u\right)\right] \\
+D_{x}\left[k^{\prime}(u) u_{t} D_{x}\left(R^{\alpha} u\right)+\right. & \left.k(u) D_{x}\left(R^{\alpha} u_{t}\right)\right]+D_{y}\left(\left[k^{\prime}(u) u_{t} D_{y}\left(R^{\alpha} u\right)+k(u) D_{y}\left(R^{\alpha} u_{t}\right)\right]\right) \\
=D_{x}\left[k^{\prime}(u) u_{t} D_{x}\left(R^{\alpha} u\right)+k(u) D_{x}\left(R^{\alpha} u_{t}\right)-D_{t}\left(k(u) D_{x} R^{\alpha} u\right)\right] & \\
& \quad+D_{y}\left[k^{\prime}(u) u_{t} D_{y}\left(R^{\alpha} u\right)+k(u) D_{y}\left(R^{\alpha} u_{t}\right)-D_{t}\left(k(u) D_{y} R^{\alpha} u\right)\right] \equiv 0
\end{aligned}
$$

because $D_{t} R^{\alpha} u=R^{\alpha} u_{t}$.

Similarly, it is easy to prove that conservation laws corresponding to $W_{2}$ and $W_{3}$ are trivial too. 
For $W_{4}$ after equivalent transformations, we find

$$
C_{4}^{t}=u, \quad C_{4}^{x}=k(u) D_{x}\left(R^{\alpha} u\right), \quad C_{4}^{y}=k(u) D_{y}\left(R^{\alpha} u\right) .
$$

The corresponding conservation law is non-trivial, and it coincides with the considered Equation (16).

For $W_{5}$, we have

$$
C_{5}^{t}=x u_{y}-y u_{x}=D_{y}(x u)-D_{x}(y u)
$$

Then,

$$
\begin{aligned}
D_{t}\left(C_{5}^{t}\right)+D_{x}\left(C_{5}^{x}\right)+D_{y}\left(C_{5}^{y}\right)=D_{t}\left[D_{y}(x u)-D_{x}(y u)\right]+D_{x}\left(C_{5}^{x}\right) & +D_{y}\left(C_{5}^{y}\right) \\
& =D_{x}\left(C_{5}^{x}-y u_{t}\right)+D_{y}\left(C_{5}^{y}+x u_{t}\right) .
\end{aligned}
$$

Now, we can replace $u_{t}$ in view of Equation (16). Since the components $C_{5}^{x}$ and $C_{5}^{y}$ do not depend on $u_{t}$, we obtain that $C^{t}=0$. After transformations, we find

$$
\begin{aligned}
& C^{x}=k\left[D_{y}\left(R^{\alpha} u\right)-D_{x}\left(R^{\alpha}\left(x u_{y}-y u_{x}\right)\right)-y D_{x}^{2}\left(R^{\alpha} u\right)+x D_{x} D_{y}\left(R^{\alpha} u\right)\right] \\
& C^{y}=k\left[-D_{x}\left(R^{\alpha} u\right)-D_{y}\left(R^{\alpha}\left(x u_{y}-y u_{x}\right)\right)+x D_{y}^{2}\left(R^{\alpha} u\right)+y D_{x} D_{y}\left(R^{\alpha} u\right)\right] .
\end{aligned}
$$

Now, let $k(u)$ be a power function: $k(u)=u^{\gamma}$. Then, Equation (16) has one additional symmetry $X_{6}$ defined by (26). For this symmetry, we have $W_{6}=-u-\gamma t u_{t}$. It can be shown that, in this case after transformations, one can get a conserved vector with the components (49).

For $k(u)=u^{-1}$, we have an infinite number of symmetries $X_{\infty}$ defined by (27). In this case, we have $W_{\infty}=-2 A_{x} u-A u_{x}-B u_{y}$. Then,

$$
C_{\infty}^{t}=W_{\infty}=-2 A_{x} u-D_{x}(A u)+A_{x} u-D_{y}(B u)+B_{y} u=-D_{x}(A u)-D_{y}(B u),
$$

since $A_{x}=B_{y}$. Thus, we have $C_{\infty}^{t}=0$ and

$$
\begin{aligned}
& C^{x}=u^{-2} u_{y}\left[A D_{y}\left(R^{\alpha} u\right)-B D_{x}\left(R^{\alpha} u\right)\right]+u^{-1}\left[D_{x}\left(R^{\alpha}\left(2 A_{x} u+A u_{x}+B u_{y}\right)\right)-A \Delta R^{\alpha} u-2 A_{x} D_{x}\left(R^{\alpha} u\right)\right], \\
& C^{y}=u^{-2} u_{x}\left[B D_{x}\left(R^{\alpha} u\right)-A D_{y}\left(R^{\alpha} u\right)\right]-u^{-1}\left[D_{y}\left(R^{\alpha}\left(2 A_{x} u+A u_{x}+B u_{y}\right)\right)+B \Delta R^{\alpha} u+2 A_{x} D_{y}\left(R^{\alpha} u\right)\right] .
\end{aligned}
$$

Thus, several different conservation laws have been found for the nonlinear space-fractional diffusion equation with the Riesz potential.

In conclusion, we note that, despite the fact that we consider nonlinear space-fractional diffusion Equation (16) only in two-dimensional space, all results for nonlinear self-adjointness and explicit forms of the Noether operators are valid for arbitrary dimension $n$. The proposed technique gives one the opportunity to construct conservation laws for a wide class of fractional differential equations with the Riesz potential.

Author Contributions: Conceptualization, S.Y.L.; methodology, S.Y.L.; software, N.S.B.; validation, S.Y.L. and N.S.B.; formal analysis, S.Y.L. and N.S.B.; investigation, S.Y.L. and N.S.B.; writing—original draft preparation, N.S.B.; writing-review and editing, S.Y.L.; supervision, S.Y.L.; project administration, S.Y.L. All authors have read and agreed to the published version of the manuscript.

Funding: This work was supported by the Ministry of Science and Higher Education of the Russian Federation (State task No. 1.3103.2017/4.6).

Conflicts of Interest: The authors declare no conflict of interest. 


\section{References}

1. Tarasov, V.E. Fractional Dynamics; Springer: Berlin, Germany, 2010.

2. Uchaikin, V.V.; Sibatov R.T. Fractional Kinetics in Space: Anomalous Transport Models; World Scientific: Hackensack, NJ, USA, 2018.

3. Aifantis, E. Fractional generalizations of gradient mechanics. In Handbook of Fractional Calculus with Applications; Tarasov, V.E., Ed.; De Gruyter: Berlin, Germany, 2019; Volume 4, pp. 241-262.

4. Biler, P.; Imbert, C.; Karch, G. Barenblatt profiles for a nonlocal porous medium equation. C. R. Math. 2011, 349, 641-645. [CrossRef]

5. Caffarelli, L.A.; Vazquez, J.L. Nonlinear porous medium flow with fractional potential pressure. Arch. Ration. Mech. Anal. 2011, 202, 537-565. [CrossRef]

6. Kilbas, A.A.; Srivastava, H.M.; Trujillo, J.J. Theory and Applications of Fractional Differential Equations; Elsevier: Amsterdam, The Netherlands, 2006.

7. Samko, S.; Kilbas, A.; Marichev, O. Fractional Integrals and Derivatives. Theory and Applications; Gordon \& Breach Sci. Publishers: London, UK, 1993.

8. Samko, S. Hypersingular Integrals and Their Applications; CRC Press: Boca Raton, FL, USA, 2001.

9. Pozrikidis, C. The Fractional Laplacian; CRC Press: Boca Raton, FL, USA, 2016.

10. Vazquez, J.L. Recent progress in the theory of nonlinear diffusion with fractional Laplacian operators. Discret. Contin. Dyn. Syst. 2014, 7, 857-885. [CrossRef]

11. Ovsyannikov, L.V. Group Analysis of Differential Equations; Academic Press: New York, NY, USA, 1982.

12. Olver, P. Applications of Lie Groups to Differential Equations; Springer: New York, NY, USA, 1986.

13. Ibragimov, N.H. Transformation Groups and Lie Algebras; World Scientific: Singapore, 2013.

14. Gazizov, R.K.; Kasatkin, A.A.; Lukashchuk, S.Y. Symmetries and group invariant solutions of fractional ordinary differential equations. In Handbook of Fractional Calculus with Applications; Fractional Differential Equations; Kochubei, A., Luchko, Y., Eds.; De Gruyter: Berlin, Germany, 2019; Volume 2, pp. 65-90.

15. Gazizov, R.K.; Kasatkin, A.A.; Lukashchuk, S.Y. Symmetries, conservation laws and group invariant solutions of fractional PDEs. In Handbook of Fractional Calculus with Applications; Fractional Differential Equations; Kochubei, A., Luchko, Y., Eds.; De Gruyter: Berlin, Germany, 2019; Volume 2, pp. 353-382.

16. Belevtsov, N.S.; Lukashchuk, S.Y. Lie group analysis of two-dimensional space-fractional model for flow in porous media. Math. Meth. Appl. Sci. 2018, 41, 9123-9133. [CrossRef]

17. Ovsyannikov, L.V. The group properties of nonlinear heat conduction equations. Dokl. Akad. Nauk SSSR 1959, 125, 492-495. (In Russian)

18. Gazizov, R.K.; Kasatkin, A.A.; Lukashchuk, S.Y. Symmetry properties of fractional diffusion equations. Phys. Scr. 2009, 136, 14-16. [CrossRef]

19. Krasilshchik, I.S.; Vinogradov, A.M. Symmetries and Conservation Laws for Differential Equations of Mathematical Physics; American Mathematical Society: Providence, RI, USA, 1999.

20. Kosmann-Schwarzbach, Y. The Noether Theorems. Invariance and Conservation Laws in the Twentieth Century; Springer: New York, NY, USA, 2011.

21. Noether, E. Invariante Variationsprobleme. Transp. Theory Stat. Phys. 1971, 1, 186-207. [CrossRef]

22. Ibragimov, N.H. Transformation Groups Applied to Mathematical Physics; Reidel: Boston, MA, USA, 1985.

23. Lukashchuk, S.Y. Constructing conservation laws for fractional-order integro-differential equations. Theor. Math. Phys. 2015, 184, 1049-1066. [CrossRef]

24. Ibragimov, N.H. A new conservation theorem. J. Math. Anal. Appl. 2007, 333, 311-328. [CrossRef]

25. Ibragimov, N.H. Nonlinear self-adjointness and conservation laws. J. Phys. A Math. Theor. 2011, 44, 432002. [CrossRef]

26. Ibragimov, N.H.; Avdonina, E.D. Nonlinear selfadjointness, conservation laws, and the construction of solutions of partial differential equations using conservation laws. Russ. Math. Surv. 2013, 68, 889-921. [CrossRef]

27. Lukashchuk, S.Y. Conservation laws for time-fractional subdiffusion and diffusion-wave equations. Nonlinear Dyn. 2015, 80, 791-802. [CrossRef]

28. Gazizov, R.K.; Ibragimov, N.H.; Lukashchuk, S.Y. Nonlinear self-adjointness, conservation laws and exact solutions of time-fractional Kompaneets equations. Commun. Nonlinear Sci. 2015, 23, 153-163. [CrossRef] 
29. Lukashchuk, S.Y. Approximate conservation laws for fractional differential equations Commun. Nonlinear Sci. 2019, 68, 147-159. [CrossRef]

30. Chirkunov, Y.A. Linear autonomy conditions for the basic Lie algebra of a system of linear differential equations. Dokl. Math. 2009, 79, 415-417. [CrossRef]

31. Rubin, B.S. One-dimensional representation, inversion, and certain properties of the Riesz potentials of radial functions. Math. Notes Acad. Sci. USSR 1983, 34, 751-757. [CrossRef]

32. Klimek, M. Stationarity-conservation laws for fractional differential equations with variable coefficients. J. Phys. A Math. Theor. 2001, 34, 6167-6184. [CrossRef]

33. Frederico, G.S.F.; Torres, D.F.M. A formulation of Noether's theorem for fractional problems of the calculus of variations. J. Math. Anal. Appl. 2007, 334, 834-846. [CrossRef]

34. Wheatcraft, S.W.; Meerschaert, M.M. Fractional conservation of mass. Adv. Water Resour. 2008, 31, $1377-1381$. [CrossRef]

(C) 2020 by the authors. Licensee MDPI, Basel, Switzerland. This article is an open access article distributed under the terms and conditions of the Creative Commons Attribution (CC BY) license (http://creativecommons.org/licenses/by/4.0/). 